\title{
Four-factor prothrombin complex concentrates: effectiveness in the reversal of anticoagulation
}

This article was published in the following Dove Press journal:

International Journal of Clinical Transfusion Medicine

4 April 2017

Number of times this article has been viewed

\section{Chakri Gavva \\ Manasa Reddy \\ Ravi Sarode}

Division of Transfusion Medicine and Hemostasis, Department of Pathology, University of Texas Southwestern Medical Center, Dallas, TX, USA
Correspondence: Ravi Sarode Department of Pathology, University of Texas Southwestern Medical Center, 5323 Harry Hines Blvd, Dallas, TX 75390, USA

Tel + I 2146334917

Fax +I 2146484067

Email ravi.sarode@utsouthwestern.edu
Abstract: Vitamin K antagonists (VKAs) continue to be the most commonly prescribed class of oral anticoagulants worldwide for the treatment and prevention of venous or arterial thromboembolic events (TEEs). While VKAs are effective at reducing the incidence of TEEs, the risk of major bleeding remains significant. In patients taking VKAs and presenting with clinically significant bleeding or undergoing an emergent procedure, a rapid, effective, and safe reversal agent is required. Available options for the reversal of VKAs include plasma, vitamin $\mathrm{K}$, 3-factor prothrombin complex concentrate (3F-PCC), or 4-factor prothrombin complex concentrate (4F-PCC). Herein, we review the growing evidence that supports using 4F-PCC over both plasma and 3F-PCC for emergent VKA reversal. We also discuss the various dosing options and safety profile of 4F-PCC. In addition, we summarize the limited data available for 4F-PCC in reversing the effects of direct oral anticoagulants and the management of other coagulopathic bleeding.

Keywords: anticoagulants, prothrombin complex concentrate, vitamin k antagonist, warfarin

\section{Introduction}

Oral anticoagulants are used extensively for the prevention and treatment of venous and arterial thrombosis. Until the recent introduction of direct oral anticoagulants (DOACs), vitamin $\mathrm{K}$ antagonists (VKAs) were the only oral anticoagulants used worldwide. VKAs, including warfarin, phenprocoumon, fluindione, and acenocoumarol, remain the most commonly used oral anticoagulants due to cost-effectiveness, well-established evidence, and the availability of specific reversal agents. ${ }^{1}$ VKAs continue to be the first-line therapy for patients with left ventricular assist devices (LVADs), mechanical valves, and antiphospholipid syndrome. ${ }^{2,3}$

The most significant complication of VKAs continues to be major bleeding. One option to emergently reverse the anticoagulant effects of VKAs is a 4-factor prothrombin complex concentrate (4F-PCC). In 2013, 4F-PCC was approved in the USA for VKA reversal in patients with major bleeding or requiring emergency invasive procedures. While 4F-PCC under the trade name of KCentra (CSL Behring GmbH, Marburg, Germany) is currently the only US Food and Drug Administration (FDA)-approved factor derivative for this purpose, 4F-PCCs have been available for years in many countries under the trade names Beriplex $\mathrm{P} / \mathrm{N}$, Confidex (CSL Behring GmbH, Marburg, Germany) and Octaplex (Octapharma, Lachen, Switzerland). 
Although VKA reversal is the primary indication, 4F-PCC is increasingly used off-label for reversal of other oral anticoagulants including direct activated factor X (FXa) inhibitors and direct thrombin inhibitors (DTIs). Its role in addressing coagulopathy in cardiac surgery and liver disease has also been explored.

This review will provide background regarding the contents and dosing of 4F-PCC, analyze existing literature on the efficacy and safety of 4F-PCC compared to 3-factor PCC (3F-PCC) or plasma for VKA reversal, and discuss the evidence regarding off-label use of 4F-PCC.

\section{VKAs: mechanism of action}

VKAs primarily inhibit vitamin $\mathrm{K}$ epoxide reductase. This leads to impaired recycling of reduced vitamin $\mathrm{K}$ resulting in decreased functional vitamin $\mathrm{K}$ dependent factors (VKDFs; factor II, factor VII, factor IX, factor X, and protein C and $\mathrm{S})$, placing the patient in an overall anticoagulated state. The international normalized ratio (INR), a value calculated from the prothrombin time (PT), is used to monitor the therapeutic effect of VKAs. Most indications for VKAs require a target INR of 2.0-3.0 (or 2.5-3.5 for patients with mechanical cardiac valves), corresponding to factor II (FII) and factor $\mathrm{X}(\mathrm{FX})$ activities of $30-15 \%{ }^{4}$

Multiple factors including dietary vitamin $\mathrm{K}$ intake, concurrent medications, or genetic variability in VKA metabolism affect the individual dose-response of VKA. This may lead to fluctuations in individual INRs with some patients having a supratherapeutic INR and an increased risk of bleeding. ${ }^{5}$ Real-life surveys suggest the incidence of major bleeding and intracranial hemorrhage to be $1.35 \%-3.4 \%$ per year and $0.4 \%-0.6 \%$ per year respectively. ${ }^{6}$ Hence, in patients with major bleeding or those undergoing an emergent surgical procedure, safe, effective, and rapid restoration of VKDFs is required. Current treatments to reverse VKAs include prothrombin complex concentrates (PCCs), plasma, and vitamin $\mathrm{K}$.

\section{PCC preparations}

PCCs are produced from large pools of cryo-depleted plasma adsorbed via ion exchange chromatography and then heat treated, precipitated, adsorbed to calcium phosphate, and lyophilized. ${ }^{7}$ They are available in activated and nonactivated forms. Nonactivated PCCs are classified as 3F-PCC or 4F-PCC. 3F-PCCs were available earlier than 4F-PCCs, but unlike 4F-PCCs which contain all VKDFs, 3F-PCCs do not contain a significant amount of factor VII (FVII) or natural anticoagulants protein $\mathrm{C}(\mathrm{PC})$ and protein
S (PS). ${ }^{7,8}$ Activated PCC (antiinhibitor coagulant complex, factor VIII Inhibitor Bypass Activity, FEIBA; Baxter, Deerfield, IL, USA), contains primarily activated FVII and likely FXa along with nonactivated FII and factor IX (FIX). It is indicated for the treatment of hemophilia A and B patients with inhibitors. ${ }^{9}$

\section{VKA reversal: 3F-PCC vs 4F-PCC}

Several studies have shown superior efficacy of 4F-PCC compared to 3F-PCC for VKA reversal, with similar safety profiles. ${ }^{10-12}$ A retrospective study published in 2016 by Mangram et al reviewed all consecutive trauma patients with coagulopathy secondary to oral anticoagulant use who were treated with either $3 \mathrm{~F}$ - or $4 \mathrm{~F}-\mathrm{PCC}$ over a 4-year period at two trauma centers. ${ }^{12}$ Efficacy was assessed by determining whether INR of $<1.5$ was achieved post-PCC administration. Successful VKA reversal was observed in $81 \%$ of the patients who were administered $4 F-P C C$ ( $n=16$ ) vs $51 \%$ of those who received 3F-PCC $(n=45)$. Voils and Baird performed a systematic review of 18 studies representing 654 patients who were administered 3F- or 4F-PCC, most commonly for intracerebral hemorrhage, urgent surgery or invasive procedure, and gastrointestinal bleeding. INR was successfully reversed to $<1.5$ in 6 out of 9 studies in the 3F-PCC group (baseline INR 3.3 to 5.1), vs 12 out of 13 studies in the 4F-PCC group (baseline INR 2.3 to $>20$ ), again suggesting that $4 \mathrm{~F}-\mathrm{PCC}$ is a more reliable reversal agent. ${ }^{10}$

A recent small, retrospective study by Al-Majzoub et al further demonstrated superior INR reversal of 4F-PCC compared to 3F-PCC in VKA-related bleeding. ${ }^{13}$ Despite the 3F-PCC group also receiving a mean 3.66 units of plasma within 4 hours of infusion (vs none in the $4 \mathrm{~F}-\mathrm{PCC}$ group), the 4F-PCC group still had more patients reaching the primary endpoint of an INR $\leq 1.3$ after treatment $(42.9 \%$ vs $83.3 \%$ ). Other outcomes such as hospital or intensive care unit (ICU) length of stay or survival to hospital discharge showed no significant differences between the two groups. ${ }^{13}$ However, the study did not compare hemostatic efficacy between the two groups. Hospital or ICU length of stay and survival to discharge can be confounded by a host of other factors.

\section{F-PCC dosing}

Manufacturer-recommended dosing of 4F-PCC is determined by the pretreatment INR and the patient's body weight (Table 1). Several studies have investigated whether fixed dosing of 4F-PCC has similar efficacy to individualized weight-based dosing. Thus far, the results appear to 
Table I Indications and dosing of 4-factor prothrombin complex concentrate for major bleeding or prior to an emergent procedure

\begin{tabular}{lll}
\hline Indications & Dosing & \\
\hline VKA reversal (manufacturer- & Pre-treatment INR & \\
recommended dosing) $^{\mathrm{a}}$ & 2.0 to $<4.0$ & $25 \mathrm{IU} / \mathrm{kg}$ \\
& $4.0-6.0$ & $35 \mathrm{IU} / \mathrm{kg}$ \\
& $>6.0$ & $50 \mathrm{IU} / \mathrm{kg}$ \\
DOAC reversal & \\
\hline
\end{tabular}

Notes: 'Based on pretreatment INR and patient's body weight up to a maximum $100 \mathrm{~kg}$. 'This is not a US FDA approved indication and should be used with caution and only as a last resort if no other reversal agents are available. This includes reversal of dabigatran, rivaroxaban, apixaban, and edoxaban. 'Up to a maximum of 5000 IU.

Abbreviations: DOAC, direct oral anticoagulant; INR, international normalized ratio; IU, international units; US FDA, United States Food and Drug Administration; VKA, vitamin $\mathrm{K}$ antagonist.

be mixed. Low fixed doses of 500 and 1000 IU have been shown to be effective in INR reversal, particularly in cases of INR $<5.0$, while in other studies, a fixed dose of 500 or 1000 IU may require additional infusions to achieve the target INR. ${ }^{14-16}$ In a retrospective review of 103 patients taking VKAs and presenting either with major bleeding or requiring an urgent invasive procedure, a fixed dose of 1000 IU 4F-PCC (based on FIX activity) led to $92.2 \%$ of patients having an INR $<2.0$ posttreatment with $83.5 \%$ of patients deemed to have an excellent clinical response. ${ }^{17}$ Another prospective, observational study compared a fixed dose of 1000 IU of 4F-PCC and a variable dosing regimen (based on body weight, pretreatment INR, and target INR) for VKA reversal in patients with clinically significant bleeding. The fixed dose group had better clinical outcomes $(96 \%$ vs $88 \%$, difference $8.27 \%, 90 \%$ confidence interval [CI]: 2.7-13.9) despite a slightly lower percentage of them reaching the target INR ( $91.7 \%$ vs $94.7 \%$, difference $2.99 \%, 90 \%$ CI: -8.6 to 2.7 ). The authors hypothesize that the shorter median time to infusion in the fixed dose group (130 vs 160 minutes, $p=0.015$ ) contributed to the improved clinical outcomes. ${ }^{18}$ Another potential dosing strategy includes using weight-based dosing but at lower overall doses (15-25 IU $/ \mathrm{kg}$ ) compared to that recommended by the manufacturer $(25-50 \mathrm{IU} / \mathrm{kg})$. One study found a reduced incidence of thromboembolic events (TEEs) with this protocol. ${ }^{19}$

Although the evidence regarding fixed dosing of 4F-PCC is promising, manufacturer-recommended weight-based dosing may be the most consistent option at this time until there is more standardization of fixed dosing. Regardless of whether fixed dosing or weight-based dosing is utilized, medical centers should have a predefined VKA reversal protocol in place to minimize delays in treatment. ${ }^{20}$

\section{F-PCC vs plasma for VKA reversal}

Historically, consensus guidelines recommended vitamin K supplemented with either plasma or PCC for the management of VKA-associated bleeding. ${ }^{21}$ However, plasma transfusion has an increased risk of transfusion associated circulatory overload, transfusion-related acute lung injury, transfusiontransmitted infectious diseases, and potentially severe allergic reactions. Furthermore, plasma requires thawing (if thawed plasma is unavailable) and $\mathrm{ABO}$ blood typing, resulting in a delay in VKA reversal. ${ }^{22,23}$

The current American College of Chest Physicians (ACCP) evidence-based guidelines on management of anticoagulant therapy recommends a 4F-PCC over plasma for the rapid reversal of anticoagulation in patients with VKAassociated major bleeding. ${ }^{24}$ This recommendation is in line with the results of a phase IIIb, multicenter, prospective, randomized noninferiority study comparing 4F-PCC (25-50 IU of FIX per kg and according to baseline INR) with plasma (10-15 $\mathrm{mL} / \mathrm{kg}$ and according to baseline INR) for urgent VKA reversal in patients with an INR $\geq 2$ and experiencing major bleeding. ${ }^{25}$ Most patients also received intravenous (IV) vitamin $\mathrm{K}$ in addition to 4F-PCC or plasma. Seventytwo percent of patients receiving 4F-PCC achieved effective hemostasis at 24 hours compared to $65 \%$ receiving plasma (difference $7.1 \%, 95 \% \mathrm{CI}$ : -5.8 to 19.9 ), establishing noninferiority of 4F-PCC. Moreover, $62.2 \%$ of patients receiving $4 F-P C C$ had an INR $\leq 1.3$ at 0.5 hours after the end of infusion, while only $9.6 \%$ in the plasma group reached the same endpoint (difference 52.6\%, 95\% CI: 39.4-65.9). Although 4F-PCC was not found to be superior compared to plasma for hemostatic efficacy at 24 hours after treatment, the trial was not powered to demonstrate superiority. Furthermore, concurrent IV vitamin $\mathrm{K}$ administration likely confounded assessment of hemostatic efficacy at 24 hours. A post hoc analysis of patients with visible and musculoskeletal bleeding showed that the 4F-PCC group had superior hemostatic efficacy at 4 hours after treatment compared to the plasma group (difference 32.6\%, 95\% CI: 4.5-60.7). In addition, no patients suffered from fluid overload or a similar cardiac event related to the administration of 4F-PCC. By comparison, plasma transfusion was thought to be related to fluid overload or a similar cardiac event in $6.4 \%$ of patients. This finding is most likely related to the significantly smaller infusion volumes in the 4F-PCC group (median $99.4 \mathrm{~mL}$ ) compared to the plasma group (median $813.5 \mathrm{~mL}$ ). Additional benefits of 4F-PCC included a shorter infusion time (median $17.0 \mathrm{~min}$ ) compared to plasma (median $148.0 \mathrm{~min}$ ). 
In a similar multicenter, randomized, phase IIIb trial of 168 patients requiring rapid VKA reversal prior to an urgent surgical or invasive procedure, 4F-PCC was found to be superior to plasma in both hemostasis (90\% vs $75 \%$, difference $14.3 \%, 95 \% \mathrm{CI}: 2.8 \%-25.8 \%$ ) as well as rapid INR reduction (55\% vs $10 \%$, difference $45.3 \%$, $95 \% \mathrm{CI}$ : $31.9 \%-56.4 \%){ }^{26}$ Three percent of patients in the 4F-PCC group experienced fluid overload or a similar cardiac event vs $13 \%$ in the plasma group. The findings from these studies are summarized in Table 2.

\section{VKA-associated intracranial hemorrhage}

Up to $36 \%$ of deaths during long term anticoagulation have been attributed to VKA-associated intracranial hemorrhage (VKA-ICH) with early hematoma expansion after hemorrhage being a major cause of mortality. ${ }^{27}$ To determine the optimal treatment of VKA-ICH, a prospective, multicenter, randomized trial of patients with VKA-ICH was recently conducted. ${ }^{27}$ Sixty-seven percent of patients $(18 / 27)$ in the $4 \mathrm{~F}-\mathrm{PCC}$ group (30 IU/kg) reached the primary endpoint of an INR $\leq 1.2$ within 3 hours of treatment initiation compared to $9 \%(2 / 23)$ in the plasma group (adjusted odds ratio 30.6 , 95\% CI: 4.7-197.9). Importantly, hematoma expansion was significantly greater in the plasma group. Five patients in the plasma group died from hematoma expansion compared to none in the 4F-PCC cohort. The trial was terminated prematurely due to safety concerns secondary to differences in hematoma expansion. Although there was no significant difference in functional outcomes at 90 days, the trial was not powered to assess this endpoint. Until larger studies suggest otherwise, the results of this study indicate 4F-PCC should be preferred over plasma in patients with VKA-ICH.

\section{VKA reversal prior to cardiac surgery}

VKAs remain the anticoagulant of choice in patients with mechanical cardiac valves or LVADs. ${ }^{2,3}$ In the past decade, an increasing number of heart transplants have been performed on patients with LVADs. ${ }^{28}$ Prior to LVAD explantation and heart transplantation, VKAs must be reversed to reduce the risk of bleeding during surgery. A single center combined retrospective and prospective study examined perioperative blood utilization in adults anticoagulated with VKAs and undergoing heart transplant. ${ }^{28}$ Prior to the availability of 4F-PCC, the standard of care for VKA reversal at the study center included plasma and vitamin K. After December 2013, their anticoagulation reversal protocol included 4F-PCC (or 3F-PCC if the patient had a history of heparin induced thrombocytopenia), vitamin $\mathrm{K}$, and desmopressin. Compared to the previous standard of care, the PCC protocol resulted in a decrease in total blood volume transfused $(2.45 \mathrm{~L}$ vs 4.20 $\mathrm{L}, p=0.037)$ and total units of blood products transfused (12 units vs 20 units, $p=0.033$ ) per patient. Interestingly, although PCC is more expensive than plasma, there was no significant difference in overall cost between the pre-PCC and post-PCC

Table 2 Phase Illb, multicenter, randomized, non-inferiority studies comparing efficacy of 4-factor prothrombin complex concentrate vs plasma for vitamin $\mathrm{K}$ antagonist reversal

\begin{tabular}{|c|c|c|}
\hline & Sarode et $\mathrm{al}^{25}$ & Goldstein et $\mathbf{a l}^{26}$ \\
\hline Study population & $\begin{array}{l}\text { Patients with VKA associated acute } \\
\text { major bleeding }\end{array}$ & $\begin{array}{l}\text { Patients receiving VKAs and requiring an } \\
\text { emergent surgical or invasive procedure }\end{array}$ \\
\hline 4F-PCC vs plasma: hemostatic efficacy ${ }^{\mathrm{a}}$ & 7I/98 (72.4\%) vs 68/I04 (65.4\%); & $78 / 87(90 \%)$ vs $6 \mathrm{I} / 8 \mathrm{I}$ (75\%); Difference I4.3\% \\
\hline (N/total) & Difference $7.1 \%$ ( $95 \% \mathrm{Cl}:-5.8$ to 19.9$)$ & (95\% Cl: 2.8 to 25.8$)$ \\
\hline 4F-PCC vs plasma: rapid INR reduction & $61 / 98(62.2 \%)$ vs $10 / 104(9.6 \%)$ & $48 / 87$ (55\%) vs $8 / 8$ I (10\%); Difference $45.3 \%$ \\
\hline$\left(\mathrm{N} /\right.$ total $^{\mathrm{b}}$ & Difference $52.6 \%$ (95\% Cl: 39.4 to 65.9$)$ & (95\% Cl: 31.9 to 56.4$)$ \\
\hline 4F-PCC vs plasma: infusion volumes $(\mathrm{mL})$ & Median: 99.4 vs 813.5 & Mean: 89.7 vs 818.7 \\
\hline 4F-PCC vs plasma: infusion times (min) & Median: 17.0 vs $\mid 48.0$ & Mean: 20.9 vs 140.7 \\
\hline $\begin{array}{l}\text { 4F-PCC vs plasma: incidence of related } \\
\text { serious adverse events ( } N / \text { total) }\end{array}$ & $2 / 103(1.9 \%)$ vs $4 / 109(3.7 \%)$ & $3 / 88(3 \%)$ vs $3 / 88(3 \%)$ \\
\hline $\begin{array}{l}\text { 4F-PCC vs plasma: incidence of TEEs } \\
(\mathrm{N} / \text { total) }\end{array}$ & $4 / 103(3.9 \%)$ vs $3 / 109(2.8 \%)$ & $6 / 88(7 \%)$ vs $7 / 88(8 \%)$ \\
\hline $\begin{array}{l}\text { 4F-PCC vs plasma: incidence of related } \\
\text { volume overload or other similar cardiac } \\
\text { events ( } \mathrm{N} / \text { total) }\end{array}$ & $0 / 103(0 \%)$ vs $7 / 109(6.4 \%)$ & $3 / 88(3 \%)$ vs II /88 (I3\%) \\
\hline
\end{tabular}

Notes: aln Sarode et al, hemostatic efficacy was assessed over a 24-hour period from the start of the infusion and determined by the Endpoint Adjudication Board based upon a predetermined hemostatic efficacy scale using objective criteria. In Goldstein et al, effective hemostasis refers to intraoperative or intraprocedural blood loss not exceeding the predicted blood loss by $50 \mathrm{~mL}$ or $30 \%$, no administration of non-study coagulation products, and surgeon assessed normal or mildly abnormal hemostasis. bINR $\leq 1.3$ after end of the infusion. Includes only treatment related TEEs in the study by Sarode et al.

Abbreviations: 4F-PCC, 4-Factor prothrombin complex concentrate; Cl, confidence interval; INR, international normalized ratio; TEEs, thromboembolic events; VKA, vitamin $\mathrm{K}$ antagonist. 
groups. The greater cost of PCC was likely offset by a reduction in transfused packed red blood cells, platelets, plasma, cryoprecipitate, and recombinant activated FVII (rFVIIa). As the sample size of the study is limited (12 patients pre-PCC and 11 patients post-PCC), the results warrant a larger cost effective analysis to determine the true difference in cost between plasma and 4F-PCC.

\section{Risk of TEEs}

As 4F-PCC contains a large concentration of procoagulant factors, the risk of TEEs remains a concern. ${ }^{23}$ These concerns are possibly related to previous case reports of patients with hemophilia B treated with 3F-PCC or hemophilia A and B with inhibitors treated with aPCC developing TEEs after administration of frequent and large doses. ${ }^{29,30}$ Frequent and large doses of PCC over many days can lead to cumulative elevated levels of FII and FX. This accumulation of FII and FX likely explains the TEEs reported in these patients. In contrast, patients receiving 4F-PCC for VKA reversal often only receive 1 dose which prevents FII and FX from reaching supra-physiologic levels. And, unlike previous preparations of PCC, 4F-PCC also contains the natural anticoagulant PC and PS.

An integrated analysis of the studies by Sarode et $\mathrm{a}^{25}$ and Goldstein et $\mathrm{a}^{26}$ reported a similar incidence of TEEs between the 4F-PCC $(14 / 191,7.3 \%)$ and plasma groups $(14 / 197$, $7.1 \%) .{ }^{31}$ However, these studies had a strict exclusion criteria and did not include certain patient groups such as those with a history of TEEs within 3 months of enrollment or history of antiphospholipid antibody. ${ }^{26,32}$ Hence, real-world studies to assess the risk of TEEs in unselected patients would be useful. In a single-center retrospective study of 113 consecutive adults who received 4F-PCC for VKA reversal, $6.2 \%$ (7/113) of patients developed TEEs within 60 days of $4 \mathrm{~F}-\mathrm{PCC}$ administration. ${ }^{33}$ Of these 7 patients, only 2 were thought to have developed a TEE directly related to 4F-PCC (1.8\%). Another real-world retrospective study of unselected patients receiving at least 1 dose of 4F-PCC found a higher incidence of TEEs compared to previous studies (11/93, 11.8\%). ${ }^{34} \mathrm{In}$ addition to the original indication for anticoagulation, many of the patients who developed TEEs in this study had other TEE risk factors including recent major surgery or heparin induced thrombocytopenia (a contraindication to 4F-PCC). It is likely the risk of TEEs is not directly related to 4F-PCC administration but to the withdrawal of anticoagulation in patients with an underlying prothrombotic condition or additional clinical risk factors for thrombosis. This concept is further supported by a recent meta-analysis that demonstrated no thrombotic events in 194 healthy volunteers $(95 \% \mathrm{CI}$ : 0\%-1.6\%) who received PCC. ${ }^{35}$ Regardless of whether 4F-PCC, plasma, or vitamin $\mathrm{K}$ is used for VKA reversal, clinicians must thoroughly determine the individualized risks and benefits of reversing anticoagulation in a patient with an underlying prothrombotic condition. Such conditions include but are not limited to a recent history of an arterial or venous TEE, severe peripheral vascular disease, disseminated intravascular coagulation, and a known hereditary or acquired thrombophilia.

\section{Vitamin K supplementation}

When clinically indicated, vitamin $\mathrm{K}$ should be given at the same time as 4F-PCC to sustain its effects because VKDFs have short half-lives. In patients with ICH and major bleeding, $10 \mathrm{mg}$ IV vitamin $\mathrm{K}$ along with 4F-PCC should be administered because these patients are unlikely to resume VKA therapy in the following 2 to 4 weeks. Other patients requiring emergent major surgery may receive $3-5 \mathrm{mg}$ IV vitamin K or 1-2 mg IV vitamin K in patients with a mechanical heart valve or LVAD. Subcutaneous route is not recommended by ACCP guidelines, and oral administration is not effective, especially in patients with gastrointestinal bleeding.

There is evidence to support that some minor invasive procedures may be safely performed without any prior correction of INR, given the low added bleeding risk of many minor invasive procedures. ${ }^{36}$ However, if preprocedure 4F-PCC is deemed necessary, a single low, fixed dose of 1000 IU 4F-PCC will often achieve this goal, without the need for concurrent vitamin K. For elective surgeries, simply holding the dose of VKA 1 day prior to the procedure and administering $3 \mathrm{mg}$ IV vitamin $\mathrm{K}$ to patients $12-18$ hours before the surgery has proven to be safe and effective. ${ }^{4}$

\section{F-PCC: reversal of dabigatran}

Dabigatran is an oral DTI indicated for the prevention and treatment of venous thromboembolism (VTE) or for stroke prophylaxis in patients with nonvalvular atrial fibrillation. ${ }^{37}$ While clinical trials point to a lower rate of intracranial bleeding with dabigatran compared to VKAs, the incidence of gastrointestinal bleeding is greater or similar depending on the dabigatran dose.$^{38}$ Hence, effective and rapid reversal of the drug may be required in certain clinical scenarios.

Recently, idarucizumab (Praxbind; Boehringer Ingelheim, Ridgefield, CT, USA), a humanized monoclonal antibody fragment, was developed for the specific neutralization of dabigatran. This product has been shown to successfully reverse the anticoagulant effect of dabigatran. ${ }^{37}$ Moreover, 
idarucizumab may be able to shorten the time to treatment and thus, improve clinical outcomes. However, idarucizumab may not be available in all medical centers, and alternative methods to control dabigatran-associated bleeding or reverse the anticoagulant effect in patients undergoing urgent surgery may be required.

Studies evaluating the reversal of dabigatran with $4 \mathrm{~F}-\mathrm{PCC}$ show variable results. At least 2 studies suggest that 4F-PCC has no significant effect on the anticoagulant action of dabigatran while other ex vivo studies demonstrate its effectiveness. ${ }^{39-41}$ Although there are no systematic clinical trials to investigate clinical reversal of dabigatran-associated bleeding with PCCs, a recently published case series by Díaz et al evaluated 5 patients who were treated with 4F-PCC for dabigatran-associated gastrointestinal bleeding. Four of the 5 patients experienced adequate control of bleeding with no TEEs reported in the 6 months after PCC administration. ${ }^{42}$

Despite the limited evidence, $50 \mathrm{IU} / \mathrm{kg}$ 4F-PCC may be considered as a last resort for urgent dabigatran reversal in centers where idarucizumab is not available. Please note, however, 4F-PCC is not US FDA approved for this indication and should be used with caution. If the patient does not have life-threatening bleeding, simply discontinuing the drug (halflife of $12-17$ hours $)^{43}$ will restore normal hemostasis $12-24$ hours after the last dose if creatinine clearance is $>80 \mathrm{~mL} / \mathrm{min}$ or 24-36 hours if creatinine clearance is $50-80 \mathrm{~mL} / \mathrm{min}^{44}$

\section{F-PCC: reversal of direct factor Xa inhibitors}

Rivaroxaban, apixaban, and edoxaban are both oral direct FXa inhibitors, approved for stroke prevention in patients with nonvalvular atrial fibrillation as well as for the treatment and prevention of VTE. ${ }^{45}$ Studies have demonstrated a lower rate of intracranial hemorrhage with rivaroxaban and high dose edoxaban (60 mg) vs VKAs but a greater incidence of gastrointestinal bleeding. In contrast, apixaban was associated with a lower rate of intracranial bleeding and similar rate of gastrointestinal bleeding compared to VKAs, while low dose edoxaban $(30 \mathrm{mg})$ was associated with a lower rate of both intracranial and gastrointestinal bleeding. ${ }^{38}$ Similar to dabigatran, normal hemostasis is expected in 12-36 hours after drug discontinuation depending on the patient's renal function. ${ }^{44}$ However, with all 3 drugs, reversal of their anticoagulant effects may be required in patients with major bleeding or those undergoing urgent procedures. Andexanet alfa (Portola Pharmaceuticals, San Francisco, CA, USA) is an antidote for both direct and indirect FXa inhibitors (low molecular weight heparin and fondaparinux), while ciraparantag (Perosphere, Danbury, CT, USA) is considered a universal reversal agent that can bind direct and indirect FXa inhibitors, DTIs, and unfractionated heparin. Neither are commercially available at this time. ${ }^{46}$ Thus currently, alternative reversal strategies of direct FXa inhibitors must be utilized.

A randomized, double-blind, placebo-controlled study with 12 healthy male volunteers found that a dose of 50 IU/ $\mathrm{kg}$ 4F-PCC immediately and completely reverses the anticoagulant effect of rivaroxaban. ${ }^{40} \mathrm{~A}$ study comparing $3 \mathrm{~F}-\mathrm{PCC}$ and $4 \mathrm{~F}-\mathrm{PCC}$ in healthy volunteers on rivaroxaban found 50 $\mathrm{IU} / \mathrm{kg}$ 4F-PCC to be more effective at reducing PT than 50 $\mathrm{IU} / \mathrm{kg}$ 3F-PCC, but 3F-PCC improved thrombin generation more than 4F-PCC did. The authors speculated that the findings may reflect the different procoagulant contents in these products, but that both 3F-PCC and 4F-PCC at least partially reverse the anticoagulant effects of rivaroxaban in healthy adults. ${ }^{47}$ Barco et al demonstrated that 37.5 IU/kg 4F-PCC resulted in partial reversal of thrombin generation and $\mathrm{PT}$ prolongation while $25 \mathrm{IU} / \mathrm{kg}$ did not. ${ }^{48}$ The same authors repeated the study with apixaban and found both doses do partially reverse apixaban, with similar increases in endogenous thrombin potential (ETP) and sustained PT restoration over a 24-hour period. These lower doses may not result in instant or complete restoration of hemostasis, however, as ETP was not immediately restored to pre-apixaban levels. ${ }^{49}$

A recent study by Zahir et al evaluated the effects of $4 \mathrm{~F}-\mathrm{PCC}$ in healthy volunteers who received a single dose of edoxaban (60 mg) and underwent a punch biopsy, a bleeding model developed by the authors. ${ }^{50} 4 \mathrm{~F}-\mathrm{PCC}$ at $50 \mathrm{IU} / \mathrm{kg}$ was able to fully restore ETP and reverse the anticoagulant effect on bleeding duration and blood loss, while 25 and $10 \mathrm{IU} / \mathrm{kg}$ had only partial and no effect respectively. Although 4F-PCC was unable to fully correct the PT, there was a dose-dependent response as $50 \mathrm{IU} / \mathrm{kg}$ had a greater reduction in PT than 25 and $10 \mathrm{IU} / \mathrm{kg}$. The results of this study are promising and lend support for the use of $50 \mathrm{IU} / \mathrm{kg} 4 \mathrm{~F}-\mathrm{PCC}$ in the emergent reversal of edoxaban.

In patients requiring the emergent reversal of direct $\mathrm{FXa}$ inhibitors, the limited data suggest $50 \mathrm{IU} / \mathrm{kg}$ 4F-PCC may be an option. Again, this use is not US FDA approved and should be employed with caution. Just as in VKA reversal, reversal of DOACs must take into account the indication for anticoagulation and risk for VTE. A patient with recent VTE, advanced heart disease, antiphospholipid syndrome, or atrial fibrillation is at higher short-term risk for thrombosis, and such risk assessment should be factored into decisions regarding the necessity and degree of anticoagulant reversal. Activated PCCs and rFVIIa are generally more prothrombotic than $4 \mathrm{~F}-\mathrm{PCC}$, and thus $4 \mathrm{~F}-\mathrm{PCC}$ is typically preferred as a nonspecific reversal agent. ${ }^{51,52}$ 


\section{Other off-label uses of PCC}

PCC administration has also been explored in settings other than anticoagulation reversal, including in patients with chronic liver disease or undergoing cardiac surgery without a history of anticoagulation.

Cappabianca et al studied the use of 3F-PCC (median $1500 \mathrm{IU})$ as first-line coagulopathy treatment after cardiopulmonary bypass, when compared with plasma. ${ }^{53} 3 \mathrm{~F}-\mathrm{PCC}$ was associated with a decreased 24-hour postoperative blood loss and lower risk of red blood cell transfusion requirements. However, patients receiving 3F-PCC had an increased risk of post-operative acute kidney injury (OR 1.44, 95\% CI: 1.02-2.05) and requirement for renal replacement therapy (OR: $3.35,95 \%$ CI: $1.13-9.90) .{ }^{53}$ Given these preliminary results, it would be interesting to also compare 4F-PCC with 3F-PCC or plasma in patients undergoing cardiopulmonary bypass without a history of VKA anticoagulation.

4F-PCC has been studied in patients with chronic liver disease. Lesmana et al performed a prospective, open-label, nonrandomized study in patients with hepatobiliary disorders and liver impairment who underwent invasive procedures. ${ }^{54}$ The primary endpoint was a change in INR after treatment with 4F-PCC with a target INR of $\leq 1.5$. Approximately $83 \%$ ( 25 of 30 patients) showed a decreased INR after 4F-PCC injection. The median INR decreased from 1.6 to $1.3(p<0.001)$. Among 9 patients with active bleeding before treatment, bleeding was controlled in 5 patients (some with multiple PCC doses). The authors concluded that 4F-PCC treatment is effective in reducing INR and preventing bleeding, but more evidence regarding the use of $4 \mathrm{~F}-\mathrm{PCC}$ in patients with chronic liver disease is needed..$^{54} \mathrm{Kwon}$ and MacLaren studied 45 adults with liver failure who had an admission INR $\geq 1.5$, required an invasive procedure, and received either plasma, 4F-PCC, or rFVIIa. 4F-PCC and rFVIIa, unlike plasma, effectively and safely reduced INR in these critically ill patients and allowed expedited interventions. ${ }^{55}$ Blood product use was significantly lower in the patients who received 4F-PCC or rFVIIa as well, but rate of bleeding was similar across groups. Both studies concluded that 4F-PCC treatment is effective in reducing INR, but more evidence is needed regarding the clinical implications in patients with chronic liver disease (ie, prevention and treatment of bleeding, survival data, etc.).

The need for attention to clinical outcomes rather than laboratory values is especially important given the phenomenon of rebalanced hemostasis seen in patients with chronic liver disease, which causes routine coagulation parameters such as PT/INR to not be truly reflective of a patient's hemostatic status. ${ }^{56} \mathrm{~A}$ study by Huang et al, reviewing 4F-PCC use in 31 patients with documented liver disease, suggested that 4F-PCC is suboptimal to correct coagulopathy and hemostasis.$^{57}$ Further studies are therefore necessary to define the utility of 4F-PCC in these patients.

\section{Conclusion}

Major bleeding continues to be a risk with oral anticoagulants. Numerous studies have demonstrated the efficacy and safety of 4F-PCC for the reversal of VKAs in patients with major bleeding and in the perioperative setting. Manufacturer-recommended dosing based on the pre-treatment INR and patient's weight should be used at this time until there is more standardization regarding fixed doses. The risks and benefits of anticoagulant reversal must be carefully considered prior to any intervention in order to minimize TEEs. Furthermore, 4F-PCC is increasingly being used for the reversal of DOACs and even in patients without a history of anticoagulation. However, it should be used only after exhausting alternative hemostatic measures as data on efficacy remains limited.

\section{Disclosure}

Ravi Sarode, MD is a Consultant for CSL Behring and Octapharma. The other authors report no conflicts of interest in this work.

\section{References}

1. Kirchhof P, Ammentorp B, Darius H, et al. Management of atrial fibrillation in seven European countries after the publication of the 2010 ESC Guidelines on atrial fibrillation: primary results of the PREvention of thromboemolic events-European Registry in Atrial Fibrillation (PREFER in AF). Europace. 2014;16(1):6-14.

2. Wigle P, Hein B, Bloomfield HE, Tubb M, Doherty M. Updated guidelines on outpatient anticoagulation. Am Fam Physician. 2013;87(8):556-566.

3. Connors JM. Anticoagulation management of left ventricular assist devices. Am J Hematol. 2015;90(2):175-178.

4. Burbury KL, Milner A, Snooks B, Jupe D, Westerman DA. Short-term warfarin reversal for elective surgery - using low-dose intravenous vitamin K: safe, reliable and convenient. Br J Haematol. 2011;154(5):626-634.

5. Kinard TN, Sarode R. Four factor prothrombin complex concentrate (human): review of the pharmacology and clinical application for vitamin K antagonist reversal. Expert Rev Cardiovasc Ther. 2014;12(4): 417-427.

6. Levi M. Epidemiology and management of bleeding in patients using vitamin K antagonists. J Thromb Haemost. 2009;7(Suppl 1):103-106.

7. Kcentra Prothrombin Complex Concentrate (Human) [package insert] CSL Behring; 2013.

8. Research $\mathrm{C}$ for $\mathrm{BE}$ and Fractionated Plasma Products - Profilnine $\mathrm{SD}$ - Factor IX Complex.

9. Research $\mathrm{C}$ for $\mathrm{BE}$ and Fractionated Plasma Products - FEIBA NF (Anti-Inhibitor Coagulant Complex).

10. Voils SA, Baird B. Systematic review: 3-factor vs 4-factor prothrombin complex concentrate for warfarin reversal: does it matter? Thromb Res. 2012;130(6):833-840.

11. Voils SA, Holder MC, Premraj S, Catlin JR, Allen BR. Comparative effectiveness of 3- vs 4-factor prothrombin complex concentrate for emergent warfarin reversal. Thromb Res. 2015;136(3):595-598. 
12. Mangram A, Oguntodu OF, Dzandu JK, et al. Is there a difference in efficacy, safety, and cost-effectiveness between 3 -factor and 4-factor prothrombin complex concentrates among trauma patients on oral anticoagulants? J Crit Care. 2016;33:252-256.

13. Al-Majzoub O, Rybak E, Reardon DP, Krause P, Connors JM. Evaluation of warfarin reversal with 4-factor prothrombin complex concentrate compared to 3-factor prothrombin complex concentrate at a tertiary academic medical center. J Emerg Med. 2016;50(1):7-13.

14. Yasaka M, Sakata T, Naritomi H, Minematsu K. Optimal dose of prothrombin complex concentrate for acute reversal of oral anticoagulation. Thromb Res. 2005;115(6):455-459.

15. Abdoellakhan RA, Miah IP, Khorsand N, Meijer K, Jellema K. Fixed Vs Variable Dosing of Prothrombin Complex Concentrate in Vitamin K Antagonist-Related Intracranial Hemorrhage: A Retrospective Analysis. Neurocrit Care. 2017;26(1):64-69.

16. van Aart L, Eijkhout HW, Kamphuis JS, et al. Individualized dosing regimen for prothrombin complex concentrate more effective than standard treatment in the reversal of oral anticoagulant therapy: An open, prospective randomized controlled trial. Thromb Res. 2006;118(3):313-320.

17. Varga C, Al-Touri S, Papadoukakis S, Caplan S, Kahn S, Blostein M. The effectiveness and safety of fixed low-dose prothrombin complex concentrates in patients requiring urgent reversal of warfarin (CME). Transfusion. 2013;53(7):1451-1458.

18. Khorsand N, Veeger NJGM, van Hest RM, Ypma PF, Heidt J, Meijer K. An observational, prospective, two-cohort comparison of a fixed vs variable dosing strategy of prothrombin complex concentrate to counteract vitamin $\mathrm{K}$ antagonists in 240 bleeding emergencies. Haematologica. 2012;97(10):1501-1506.

19. Manuel F, Zemrak W, Smith K, et al. Low-dose four-factor prothrombin complex concentrate reduces harmful thromboembolic events. Crit Care Med. 2016;44(12):122.

20. Khorsand N, Kooistra HA, Van Hest RM, Veeger NJ, Meijer K. A systematic review of prothrombin complex concentrate dosing strategies to reverse vitamin K antagonist therapy. Thromb Res. 2015;135(1):9-19.

21. Ansell J, Hirsh J, Hylek E, Jacobson A, Crowther M, Palareti G. Pharmacology and management of the vitamin $\mathrm{K}$ antagonists : American College of Chest Physicians Evidence-Based Clinical Practice Guidelines (8th Edition). Chest. 2008;133(6 Suppl):160S-198S.

22. McLeod BC. Therapeutic apheresis: use of human serum albumin, fresh frozen plasma and cryosupernatant plasma in therapeutic plasma exchange. Best Pract Res Clin Haematol. 2006;19(1):157-167.

23. Yates SG, Sarode R. New strategies for effective treatment of vitamin $K$ antagonist-associated bleeding. J Thromb Haemost. 2015;13(Suppl 1): S180-S186.

24. Holbrook A, Schulman S, Witt DM, et al. Evidence-based management of anticoagulant therapy: antithrombotic therapy and prevention of thrombosis, 9th ed: American College of Chest Physicians Evidence-Based Clinical Practice Guidelines. Chest. 2012;141(2 Suppl):e152S-e184S.

25. Sarode R, Milling TJ Jr, Refaai MA, et al. Efficacy and safety of a 4-factor prothrombin complex concentrate in patients on vitamin $\mathrm{K}$ antagonists presenting with major bleeding: a randomized, plasmacontrolled, phase IIIb study. Circulation. 2013;128(11):1234-1243.

26. Goldstein JN, Refaai MA, Milling TJ Jr, et al. Four-factor prothrombin complex concentrate vs plasma for rapid vitamin $\mathrm{K}$ antagonist reversal in patients needing urgent surgical or invasive interventions: A phase 3b, open-label, non-inferiority, randomised trial. Lancet. 2015;385(9982):2077-2087.

27. Steiner T, Poli S, Griebe M, et al. Fresh frozen plasma vs prothrombin complex concentrate in patients with intracranial haemorrhage related to vitamin $\mathrm{K}$ antagonists (INCH): a randomised trial. Lancet Neurol. 2016;15(6):566-573.

28. Pratt Cleary J, Hodge L, Palmer B, Barreiro CJ, Ingemi A. 4-factor prothrombin complex concentrate $\left(\mathrm{PCC} 4, \mathrm{Kcentra}^{\circledR}\right)$ protocol reduces blood requirements for heart transplantation: a novel protocol. Ann Transplant. 2016;21:531-537.

29. Fuerth JH, Mahrer P. Myocardial infarction after factor IX therapy. JAMA. 1981;245(14):1455-1456.
30. Grupo R, Bove K, Donaldson VH. Fatal myocardial necrosis associated with prothrombin-complex concentrate therapy in hemophilia A. NEngl J Med. 1983;309(4):242-243.

31. Milling TJ Jr, Refaai MA, Sarode R, et al. Safety of a 4-factor prothrombin complex concentrate vs plasma for vitamin K antagonist reversal: an integrated analysis of two phase IIIb clinical trials. Acad Emerg Med. 2016;23(4):466-475.

32. Sarode R. Four-factor prothrombin complex concentrate vs plasma for urgent vitamin K antagonist reversal: new evidence. Clin Lab Med. 2014;34(3):613-621.

33. Joseph R, Burner J, Yates S, Strickland A, Tharpe W, Sarode R. Thromboembolic outcomes after use of a four-factor prothrombin complex concentrate for Vitamin $\mathrm{K}$ antagonist reversal in a real-world setting. Transfusion. 2016;56(4):799-807.

34. Sin J, Berger K, Lesch, C. Four-factor prothrombin complex concentrate for life-threatening bleeds or emergent surgery. Crit Care Med. 2015;43(12 Suppl 1):1-2.

35. Barco S, Picchi C, Trinchero A, Middeldorp S, Coppens M. Safety of prothrombin complex concentrate in healthy subjects. Br J Haematol. 2016;41(13):3-5.

36. Puchalski JT, Argento AC, Murphy TE, Araujo KL, Pisani MA. The safety of thoracentesis in patients with uncorrected bleeding risk. Ann Am Thorac Soc. 2013;10(4):336-341.

37. Pollack CV, Reilly Pa, Eikelboom J, et al. Idarucizumab for dabigatran reversal. N Engl J Med. 2015;373(6):511-520.

38. Eikelboom J, Merli G. Bleeding with direct oral anticoagulants vs warfarin : clinical experience. Am J Med. 2016;129(11):S33-S40.

39. Lindahl TL, Wallstedt M, Gustafsson KM, Persson E, Hillarp A. More efficient reversal of dabigatran inhibition of coagulation by activated prothrombin complex concentrate or recombinant factor VIIa than by four-factor prothrombin complex concentrate. Thromb Res. 2015;135(3):544-547.

40. Eerenberg ES, Kamphuisen PW, Sijpkens MK, Meijers JC, Buller HR, Levi M. Reversal of rivaroxaban and dabigatran by prothrombin complex concentrate: a randomized, placebo-controlled, crossover study in healthy subjects. Circulation. 2011;124(14):1573-1580.

41. Grottke O, Aisenberg J, Bernstein R, et al. Efficacy of prothrombin complex concentrates for the emergency reversal of dabigatran-induced anticoagulation. Crit Care. 2016;20(1):115.

42. Díaz MQ, Borobia AM, Núñez MA, et al. Use of prothrombin complex concentrates for urgent reversal of dabigatran in the Emergency Department. Haematologica. 2013;98(11):e143-e144.

43. Connolly S, Ezekowitz M, Yusuf S, et al; RE-LY Steering Committee and Investigators. Dabigatran vs warfarin in patient with atrial fibrillation. N Engl J Med. 2009;361(12):1139-1151.

44. Levi M. Management of bleeding in patients treated with direct oral anticoagulants. Crit Care. 2016;20:249.

45. Adcock DM, Gosselin R. Direct oral anticoagulants (DOACs ) in the laboratory : 2015 Review. Thromb Res. 2015;136(1):7-12.

46. Hu TY, Vaidya VR, Asirvatham SJ. Reversing anticoagulant effects of novel oral anticoagulants : role of ciraparantag, andexanet alfa, and idarucizumab. Vascu Health Risk Manag. 2016;12:35-44.

47. Levi M, Moore KT, Castillejos CF, et al. Comparison of three-factor and four-factor prothrombin complex concentrates regarding reversal of the anticoagulant effects of rivaroxaban in healthy volunteers. JThromb Haemost. 2014;12(9):1428-1436.

48. Barco S, Whitney Cheung Y, Coppens M, Hutten BA, Meijers JC, Middeldorp $S$. In vivo reversal of the anticoagulant effect of rivaroxaban with four-factor prothrombin complex concentrate. Br J Haematol. 2016;172(2):255-261.

49. Cheung YW, Barco S, Hutten BA, Meijers JC, Middeldorp S, Coppens $M$. In vivo increase in thrombin generation by four-factor prothrombin complex concentrate in apixaban-treated healthy volunteers. J Thromb Haemost. 2015;13(10):1799-1805.

50. Zahir H, Brown KS, Vandell AG, et al. Edoxaban effects on bleeding following punch biopsy and reversal by a 4-factor prothrombin complex concentrate. Circulation. 2015;131(1):82-90.

51. Miesbach W, Seifried E. New direct oral anticoagulants-current therapeutic options and treatment recommendations for bleeding complications. Thromb Haemost. 2012;108(4):625-632. 
52. Levi M, Levy JH, Andersen HF, TruloffD. Safety of recombinant activated factor VII in randomized clinical trials. N Engl J Med. 2010;363(19): 1791-1800.

53. Cappabianca G, Mariscalco G, Biancari F, et al. Safety and efficacy of prothrombin complex concentrate as first-line treatment in bleeding after cardiac surgery. Crit Care. 2016;20(1):5.

54. Lesmana CR, Cahyadinata L, Pakasi LS, Lesmana LA. Efficacy of prothrombin complex concentrate treatment in patients with liver coagulopathy who underwent various invasive hepatobiliary and gastrointestinal procedures. Case Rep Gastroenterol. 2016;10(2):315-322.
55. Kwon JO, Maclaren R. Comparison of Fresh-Frozen Plasma, Four-Factor Prothrombin Complex Concentrates, and Recombinant Factor VIIa to facilitate procedures in critically Ill patients with coagulopathy from liver disease : a retrospective cohort study. Pharmacotherapy. 2016; 36(10):1047-1054.

56. Lisman T, Porte RJ. Rebalanced hemostasis in patients with liver disease: evidence and clinical consequences. Blood. 2010;116(6):878-885.

57. Huang W-T, Cang WC, Derry KL, Lane JR, von Drygalski A. Fourfactor prothrombin complex concentrate for coagulopathy reversal in patients with liver disease. Clin Appl Thromb Hemost. Epub 2016 Sept 14.
International Journal of Clinical Transfusion Medicine

\section{Publish your work in this journal}

International Journal of Clinical Transfusion Medicine is an international, peer-reviewed, open access, online journal publishing clinicalexperimental, policy-making and evidence-based practices of all topics pertaining to clinical transfusion medicine. Original research, short reports, reviews, case reports and commentaries are invited.

\section{Dovepress}

The manuscript management system is completely online and includes a very quick and fair peer-review system, which is all easy to use. Visit http://www.dovepress.com/testimonials.php to read real quotes from published authors.

Submit your manuscript here: https://www.dovepress.com/international-journal-of-clinical-transfusion-medicine-journal 